\title{
AIP
}

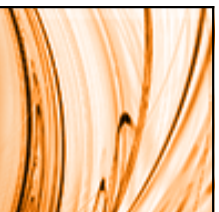

\section{Structure-performance relationships for cantilever-type piezoelectric energy harvesters}

Kyung-Hoon Cho, Hwi-Yeol Park, Jin S. Heo, and Shashank Priya

Citation: Journal of Applied Physics 115, 204108 (2014); doi: 10.1063/1.4879876

View online: http://dx.doi.org/10.1063/1.4879876

View Table of Contents: http://scitation.aip.org/content/aip/journal/jap/115/20?ver=pdfcov

Published by the AIP Publishing

\section{Articles you may be interested in}

Rotational piezoelectric wind energy harvesting using impact-induced resonance

Appl. Phys. Lett. 105, 053901 (2014); 10.1063/1.4887481

A wideband vibration energy harvester based on a folded asymmetric gapped cantilever

Appl. Phys. Lett. 104, 053902 (2014); 10.1063/1.4863923

Energy harvesting from ambient low-frequency magnetic field using magneto-mechano-electric composite cantilever

Appl. Phys. Lett. 104, 032908 (2014); 10.1063/1.4862876

Nonlinear output properties of cantilever driving low frequency piezoelectric energy harvester

Appl. Phys. Lett. 101, 223503 (2012); 10.1063/1.4768219

Cantilever driving low frequency piezoelectric energy harvester using single crystal material

$0.71 \mathrm{~Pb}(\mathrm{Mg} 1 / 3 \mathrm{Nb} 2 / 3) \mathrm{O} 3-0.29 \mathrm{PbTiO} 3$

Appl. Phys. Lett. 101, 033502 (2012); 10.1063/1.4737170

MIT LINCOLN

LABORATORY CAREERS

Discover the satisfaction of innovation and service

to the nation
- Space Control

- Air \& Missile Defense

- Communications Systems \& Cyber Security

- Intelligence, Surveillance and

Reconnaissance Systems

- Advanced
Electronics
- Tactical Systems
- Homeland
Protection
- Air Traffic Control

LINCOLN LABORATORY

MassachusetTs Institute of TeChNOLOgY

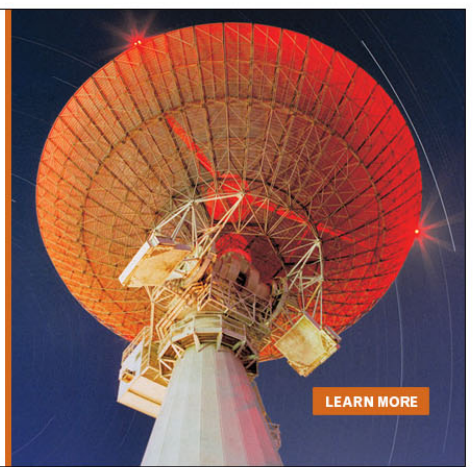




\title{
Structure-performance relationships for cantilever-type piezoelectric energy harvesters
}

\author{
Kyung-Hoon Cho, ${ }^{1, a)}$ Hwi-Yeol Park, ${ }^{1}$ Jin S. Heo, ${ }^{1}$ and Shashank Priya ${ }^{2, a)}$ \\ ${ }^{1}$ Samsung Advanced Institute of Technology, Samsung Electronics, Yongin 446-712, South Korea \\ ${ }^{2}$ Center for Energy Harvesting Materials and Systems (CEHMS), Virginia Tech, Virginia 24061, USA
}

(Received 9 March 2014; accepted 14 May 2014; published online 30 May 2014)

\begin{abstract}
This study provides comprehensive analysis of the structure-performance relationships in cantilever-type piezoelectric energy harvesters. It provides full understanding of the effect of all the practical global control variables on the harvester performance. The control variables considered for the analysis were material parameters, areal and volumetric dimensions, and configuration of the inactive and active layers. Experimentally, the output power density of the harvester was maximum when the shape of the beam was close to a square for a constant bending stiffness and a fixed beam area. Through analytical modeling of the effective stiffness for the piezoelectric bimorph, the conditions for enhancing the bending stiffness within the same beam volume as that of a conventional bimorph were identified. The harvester configuration with beam aspect ratio of 0.86 utilizing distributed inactive layers exhibited an giant output power of $52.5 \mathrm{~mW}$ and power density of $28.5 \mathrm{~mW} \mathrm{~cm}^{-3}$ at $30 \mathrm{~Hz}$ under $6.9 \mathrm{~m} \mathrm{~s}^{-2}$ excitation. The analysis further indicates that the trend in the output power with varying damping ratio is dissimilar to that of the efficiency. In order to realize best performance, the harvester should be designed with respect to maximizing the magnitude of output power. (C) 2014 AIP Publishing LLC. [http://dx.doi.org/10.1063/1.4879876]
\end{abstract}

\section{INTRODUCTION}

The abundance of vibration sources, such as machines, vehicles, roads, buildings, and the human body, has provided significant opportunity towards harvesting wasted mechanical energy for powering the distributed wireless sensor nodes. ${ }^{1-8}$ In some scenarios such as embedded systems in the wall and remote structures, the harvester is not an option rather a critical requirement. In literature, the emphasis in converting mechanical-to-electrical energy has been on three different types of transduction mechanisms: piezoelectric, electromagnetic, and electrostatic. Among these three options, piezoelectric transduction has been intensively investigated because of its inherent high energy density, structural simplicity, and better performance at reduced dimensions. Among piezoelectric harvesters, a cantilever-type bending structure has been most frequently investigated. ${ }^{9-14}$

The performance of a piezoelectric energy harvester has often been reported in terms of power density (output power per harvester volume, unit: $\mathrm{W} \mathrm{cm}^{-3}$ ) measured at a given frequency and acceleration of vibration. The reported power density values for piezoelectric energy harvesters range from $\mu \mathrm{W} \mathrm{cm}{ }^{-3}$ to a few $\mathrm{mW} \mathrm{cm}{ }^{-3} \cdot{ }^{15,16}$ Since there is no standard method of evaluating the harvester volume, it is quite difficult to make an accurate comparison in terms of the power density values. The problem arises from the fact that some researchers have used the total harvester volume including the piezoelectric layers, substrate or shim layer, and tip mass in their calculations while others have used only the beam volume including the piezoelectric layers and substrate layer.

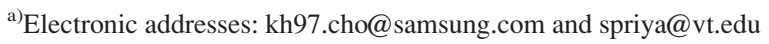

Some researchers have considered just the volume of the piezoelectric layer. However, from the viewpoint of practical applications, it is desirable to consider the total harvester volume for evaluating the power density and we encourage the community to consider this suggestion for relevant reporting and standardization.

In order to enhance the power density, the harvester should effectively absorb the mechanical energy from the vibration sources and convert it in to electrical energy. In addition, the converted electrical energy should be efficiently extracted from the harvester to be stored in a battery or supercapacitor. In some scenarios, the harvester energy could be used directly to power the sensors. For the former, many design studies have been conducted, such as topology optimization of the piezoelectric and elastic layers, ${ }^{17,18}$ shape design of the piezoelectric layer, ${ }^{19}$ control of the mass ratio between the beam and tip mass and control of the effective bending stiffness of the beam to optimize the electromechanical coupling coefficient, ${ }^{20}$ and location and length optimization of the piezoelectric layer. ${ }^{21}$ For the latter, energy harvesting circuit topologies such as synchronized switch harvesting on inductor devices and adaptive maximum power point tracking circuits have been developed. ${ }^{22-24}$ However, one fundamental area that has not been addressed in detail in literature is related to the effect of the dimensions and layering configuration of the piezoelectric cantilever beam on the output power density at a fixed frequency and acceleration.

In this study, we investigate the design of cantilevertype piezoelectric energy harvester in terms of the material parameters, thickness, and dimensions of the shim and piezoelectric layers. The results provide firm guidance on the structure-performance relationships illustrating the effect of 
the global parametric variation on the power density of the harvester. Moreover, we propose a structural design of the piezoelectric cantilever beam that utilizes the distributed inactive layers in order to further increase the output power. The analysis considers the elastic characteristics of the piezoelectric bimorph composites and proposes a novel method for increasing the equivalent mass (and consequently the output power) without changing the dimensions of the beam through control of the bending stiffness.

\section{CONTROL OF DIMENSIONS OF HARVESTER BEAM}

We consider a cantilever beam structure, shown in Fig. 1, consisting of two active piezoelectric layers, a central inactive shim layer and epoxy bonding layers. Under constant vibration conditions, we can estimate the maximum power of the piezoelectric cantilever by using Eq. (1) derived from a lumped parameter model ${ }^{25}$

$$
P=\frac{m_{e q} Y^{2} \omega_{n}^{3}}{4 \zeta},
$$

where $m_{e q}$ is the equivalent mass, $Y$ is the displacement of the cantilever base, $\omega_{n}$ is the natural frequency of the cantilever, and $\zeta$ is the damping ratio. By considering the geometric configuration of the piezoelectric cantilever beam in Fig. 1 and rewriting Eq. (1) in terms of the dimensions of the beam, the maximum power can be expressed as

$$
\begin{gathered}
P=\frac{a^{2} \sqrt{m_{e q}^{3} L^{3}}}{4 \zeta \sqrt{3 E I}}, \\
E I=\frac{2 b}{3}\left[c_{I} \frac{t_{I}^{3}}{8}+c_{A}\left(\left(t_{A}+t_{E}+\frac{t_{I}}{2}\right)^{3}-\left(t_{E}+\frac{t_{I}}{2}\right)^{3}\right)\right. \\
\left.+c_{E}\left(\left(t_{E}+\frac{t_{I}}{2}\right)^{3}-\frac{t_{I}^{3}}{8}\right)\right],
\end{gathered}
$$

where $a$ is the acceleration of the vibration, $L$ is the length of the beam, $E I$ is the bending stiffness of the beam, $b$ is the width of the beam, and $c_{I}, c_{A}$, and $c_{E}$ and $t_{I}, t_{A}$, and $t_{E}$ are the elastic moduli and thicknesses of the shim, piezoelectric, and epoxy bonding layers, respectively. The relevant control variables are $m_{e q}, L, b, c_{I}, c_{A}, t_{I}$, and $t_{A}$ when the frequency and

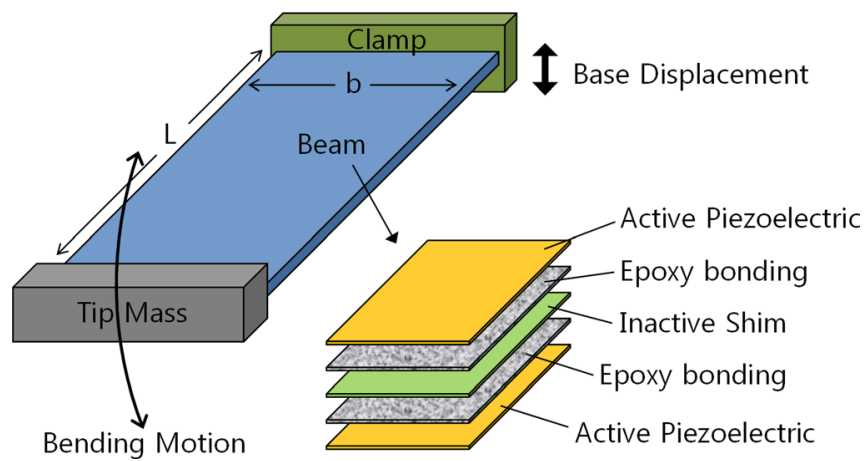

FIG. 1. Schematic diagram of cantilever-type piezoelectric energy harvester and its layer configuration. acceleration are fixed. The parameter $\zeta$ is not a practical control variable, since it is dependent upon the complex interaction occurring between the material characteristics and structural configuration. In this study, the cantilever beams with lengths longer than $10 \mathrm{~mm}$ were considered and all beams were composed of the same materials, so the effect of airflow or squeeze damping were not significant and the differences in $\zeta$ were minimized. ${ }^{26}$

We selected a piezoelectric layer with a $t_{A}$ of $130 \mu \mathrm{m}$ and a $c_{A}$ of $66 \mathrm{GPa}$ (PZT: PSI-5A4E, Piezo Systems, Inc.) and a common spring steel with a high elastic modulus of $210 \mathrm{GPa}$ as an inactive shim layer (SUS304H, JINEI Corp.). The stiffness coupling parameter of the beam $\left(\kappa_{E I}\right)$ is an important factor affecting the electromechanical coupling coefficient of the harvester device and is represented as ${ }^{20}$

$$
\kappa_{E I}=\frac{\left(1+\frac{t_{I}}{t_{A}}\right)^{2}}{\frac{1}{6}\left(\frac{c_{I}}{c_{A}}\right)\left(\frac{t_{I}}{t_{A}}\right)^{3}+\frac{1}{3}+\left(1+\frac{t_{I}}{t_{A}}\right)^{2}} .
$$

When the elastic modulus values of piezoelectric and shim layers are known, an optimum thickness ratio between the piezoelectric and shim layers can be obtained by finding the maximum $\kappa_{E I}$ position in the $\kappa_{E I}$ vs. $t_{I} / t_{A}$ curve, as shown in Fig. 2. The optimum $t_{I} / t_{A}$ value is calculated as

$$
\begin{aligned}
\left(\frac{t_{I}}{t_{A}}\right)_{o p t}= & \left\{\left[\left(\frac{2}{c_{I} / c_{A}}-1\right)^{2}-1\right]^{1 / 2}+\frac{2}{c_{I} / c_{A}}-1\right\}^{-1 / 3} \\
& +\left\{\left[\left(\frac{2}{c_{I} / c_{A}}-1\right)^{2}-1\right]^{1 / 2}+\frac{2}{c_{I} / c_{A}}-1\right\}^{1 / 3}-1
\end{aligned}
$$

and was found to be 0.6 in our case. Therefore, we decided to use an $80 \mu$ m-thick SUS304H plate as a shim layer.

To control the $m_{e q}, L$, and $b$ of the beam, we utilized a finite element analysis tool (ANSYS) to predict the fundamental frequency of the structure with respect to the specific

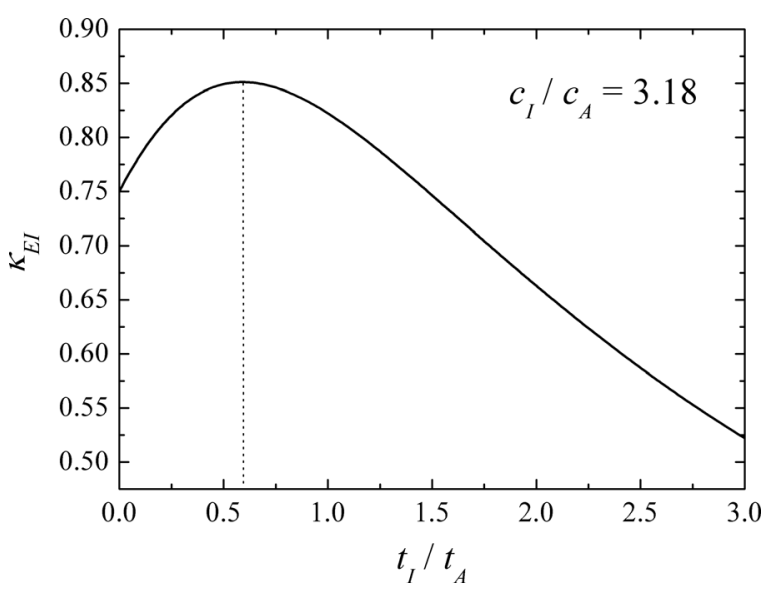

FIG. 2. Stiffness coupling parameter of cantilever beam as a function of thickness ratio between the shim and piezoelectric layers when the elastic modulus ratio between the shim and piezoelectric layers is 3.18 . 
(a)
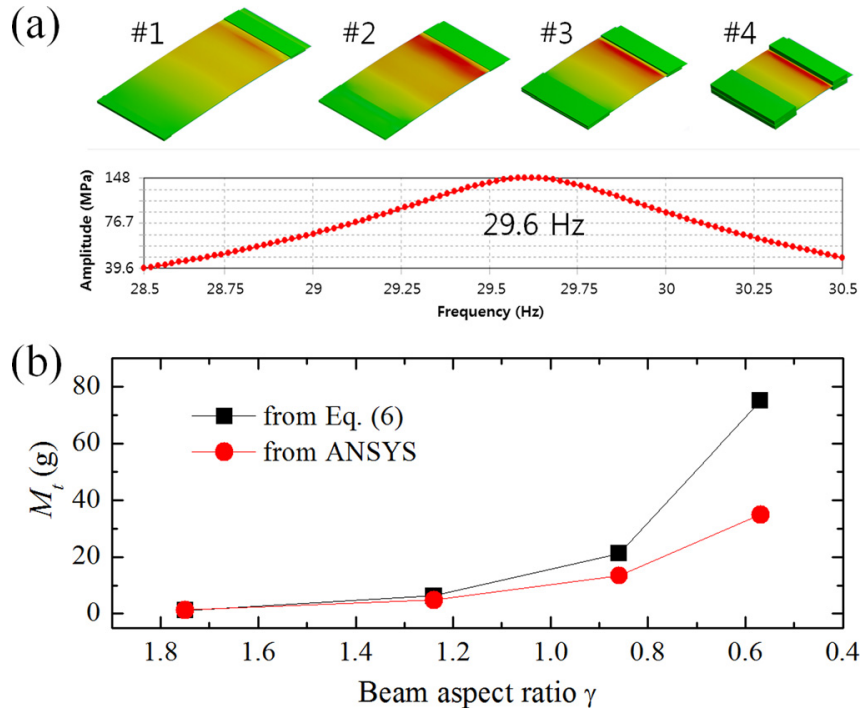

FIG. 3. (a) Harvester beam structures designed using ANSYS with various aspect ratios for a fundamental frequency of $29.6 \mathrm{~Hz}$ and (b) calculated and simulated tip mass values of the harvester beam as a function of beam aspect ratio.

dimensions of the beam and tip mass, as shown in Fig. 3(a). Our target vibration frequency range was $25-35 \mathrm{~Hz}$ as this meets the requirements for most of the common vibration platforms. The initial input values of $m_{e q}, L$, and $b$ were based on the natural frequency expression of the cantilever beam for the first bending mode ${ }^{25}$

$$
\omega_{n}=\sqrt{\frac{k_{e q}}{m_{e q}}}=\sqrt{\frac{3 E I / L^{3}}{(33 / 140) m L+M_{t}}},
$$

where $k_{e q}$ is the equivalent spring constant, $m$ is the mass per unit beam length, and $M_{t}$ is the tip mass. The $M_{t}$ value was then precisely controlled in ANSYS to make the fundamental frequency approach $29.6 \mathrm{~Hz}$ (center point of the desired range). First, the aspect ratio $(\gamma)$ of the beam was varied while the bending stiffness was fixed (i.e., $L$ is varied and $b$ is fixed). As seen in Fig. 3(b), the tip mass required for a fundamental frequency of $29.6 \mathrm{~Hz}$ increases with decreasing beam aspect ratio. However, a large discrepancy between the tip mass value calculated from Eq. (6) and that obtained from the ANSYS simulation was observed when the aspect ratio was decreased below 1. Equation (6) is useful for estimating the dimensions and tip mass required for a target fundamental frequency when the length of the beam is sufficiently long. However, it was found to be inappropriate for obtaining practical design parameters when the length is too short and the tip mass is large. The deviation of the calculated tip mass value from the simulated value might occur because the lumped parameter model assumes that the tip mass is a point mass. Next, we modified the aspect ratio of the beam while fixing the surface area of the beam with an $L$ of $31 \mathrm{~mm}$ and a $b$ of $36 \mathrm{~mm}$ in order to confirm the effect of the aspect ratio when the piezoelectric active layer volume is fixed. All design parameters of the cantilever beams (samples \#1 through \#6) extracted from the simulation are summarized in Table I.
TABLE I. Design parameters of the harvester beam extracted from the ANSYS simulation.

\begin{tabular}{lcccccc}
\hline \hline & $L(\mathrm{~mm})$ & $b(\mathrm{~mm})$ & $\gamma$ & $M_{t}(\mathrm{~g})$ & $E I\left(\mathrm{Nm}^{2}\right)$ & $f_{n}(\mathrm{~Hz})$ \\
\hline$\# 1$ & 63.2 & 36 & 1.75 & 1.29 & 0.00752 & 29.6 \\
$\# 2$ & 44.5 & 36 & 1.24 & 4.88 & 0.00752 & 29.6 \\
$\# 3$ & 31 & 36 & 0.86 & 13.48 & 0.00752 & 29.6 \\
$\# 4$ & 20.5 & 36 & 0.57 & 34.98 & 0.00752 & 29.6 \\
$\# 5$ & 47 & 23.7 & 1.98 & 3.126 & 0.00496 & 29.7 \\
$\# 6$ & 21.5 & 51.9 & 0.41 & 48.8 & 0.01085 & 29.8 \\
\hline \hline
\end{tabular}

Based on the simulated parameters, we fabricated the harvester configurations and evaluated their power generation performance. The shim layer was sandwiched by two PZT layers poled in the thickness direction using epoxy (DP$460,3 \mathrm{M})$. A homemade pressing tool was utilized to achieve a homogeneous thickness of the laminate beam, and an epoxy bonding layer that was around $10 \mu \mathrm{m}$ thick was formed after the curing process at $80^{\circ} \mathrm{C}$. Tungsten was used as a tip mass material for the cantilevers. The cantilevers were placed on a vibration exciter (type 4809, Brüel \& Kjær), and the electrodes of the parallel-type piezoelectric bimorph were connected to an oscilloscope (WavePro 715Zi, LeCroy) with a load resistance for power monitoring. The acceleration magnitude of the vibration was controlled so that it was always $6.9 \mathrm{~m} \mathrm{~s}^{-2}$ (rms) during the measurement. The maximum power values were obtained at the resonance frequency of the cantilevers under an optimum load resistance.

Figure 4 shows the natural frequency, equivalent mass, simulated stress on the surface of the piezoelectric layer, output power, and power density for harvester samples \#1 through \#4 (see Table I for sample information). The natural frequencies of the fabricated harvesters were near $29 \mathrm{~Hz}$, which corresponds well with the simulated values. The equivalent mass was found to exponentially increase with the decreasing beam aspect ratio (Fig. 4(b)). As shown in

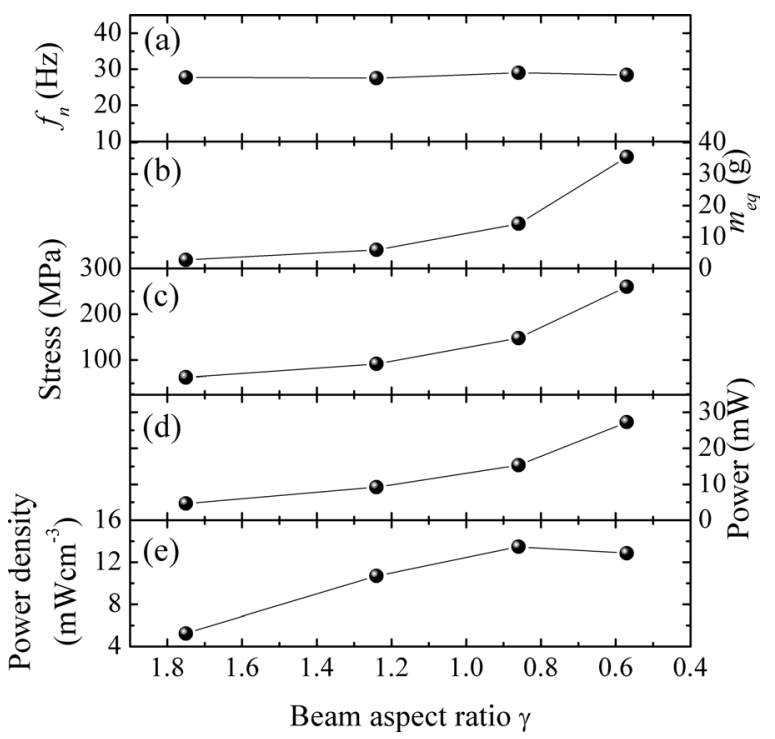

FIG. 4. Properties of harvester samples \#1 through \#4: (a) measured fundamental frequency, (b) equivalent mass, (c) simulated stress applied on the piezoelectric layer, (d) measured output power, and (e) measured power density. 
Fig. 4(c), we analyzed the stress generated on the piezoelectric layer with an acceleration condition of $9.8 \mathrm{~m} \mathrm{~s}^{-2}(1 \mathrm{G}$, commonly used nomenclature in literature) using ANSYS and found that the average stress exponentially increased similar to that of the equivalent mass. Consequently, during the bending of the beam, an increase in the equivalent mass and a decrease in the aspect ratio enhance the stress applied to the surface of the piezoelectric layer, resulting in an exponential increase in output power (from $4.69 \mathrm{~mW}$ for sample \#1 to $27.29 \mathrm{~mW}$ for sample \#4), as shown in Fig. 4(d). However, the power density, an important factor for choosing the total volume of a device within which a certain number of harvesters can be integrated, showed a maximum value of 13.47 $\mathrm{mW} \mathrm{cm}{ }^{-3}$ for the beam aspect ratio of 0.86 (sample \#3) and decreased again with further decrease in the aspect ratio down to 0.57 . We employed the high-density $\left(19.25 \mathrm{~g} \mathrm{~cm}^{-3}\right)$ tungsten to minimize the tip mass volume. Considering the drastic increase in tip mass and decrease in power density, a beam with a small aspect ratio is especially undesirable from the viewpoint of fabrication cost and device integration. Furthermore, a heavy tip mass and the resultant large stress on the beam can be problematic from the viewpoint of the durability of the device, since the practical tensile strength of the piezoelectric ceramic is around $100 \mathrm{MPa}$. In our experiments, a crack was formed in the piezoelectric layer of sample \#4 during excitation under $1 \mathrm{G}$ acceleration, resulting in significant output power degradation. The results for samples \#5 and \#6, which have the same piezoelectric layer volumes as sample \#3, were similar, as shown in Fig. 5. The output power was significantly increased up to $36.35 \mathrm{~mW}$ for sample \#6, which has a small aspect ratio of 0.41 , but the power density was less than that of sample \#3 and its tip mass was large, $48.8 \mathrm{~g}$. The output characteristics of all harvester samples are summarized in Table II. The harvester with a beam aspect ratio of 0.86 exhibited an excellent output power density of $13.47 \mathrm{~mW} \mathrm{~cm}^{-3}$ under excitation at $6.9 \mathrm{~m} \mathrm{~s}^{-2}$ and $29 \mathrm{~Hz}$. This high power density is believed to be due to the optimum

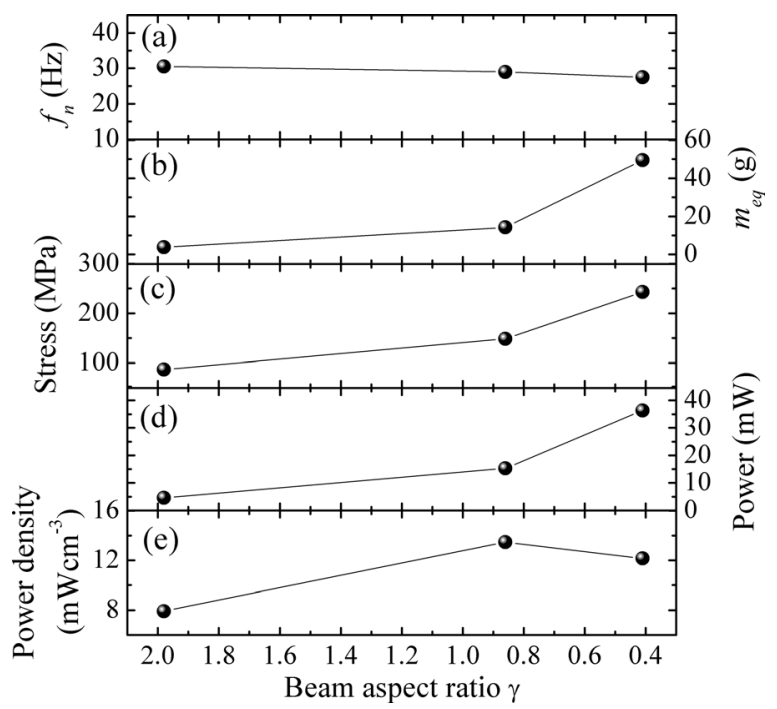

FIG. 5. Properties of harvester samples \#3, \#5, and \#6: (a) measured fundamental frequency, (b) equivalent mass, (c) simulated stress applied on the piezoelectric layer, (d) measured output power, and (e) measured power density.
TABLE II. Closed-circuit voltage $\left(\mathrm{V}_{\mathrm{CC}}\right)$, load resistance $\left(\mathrm{R}_{\mathrm{L}}\right)$, total volume, output power, and power density of the harvesters.

\begin{tabular}{lrccrc}
\hline \hline & $\begin{array}{c}\mathrm{V}_{\mathrm{CC}} \\
(\mathrm{V})\end{array}$ & $\begin{array}{c}\mathrm{R}_{\mathrm{L}} \\
(\mathrm{k} \Omega)\end{array}$ & $\begin{array}{c}\text { Volume } \\
\left(\mathrm{cm}^{3}\right)\end{array}$ & $\begin{array}{c}\text { Power } \\
(\mathrm{mW})\end{array}$ & $\begin{array}{c}\text { Power density } \\
\left(\mathrm{mW} \mathrm{cm}^{-3}\right)\end{array}$ \\
\hline$\# 1$ & 6.46 & 8.90 & 0.89 & 4.69 & 5.25 \\
$\# 2$ & 11.23 & 13.7 & 0.86 & 9.21 & 10.71 \\
$\# 3$ & 17.23 & 19.4 & 1.14 & 15.30 & 13.47 \\
$\# 4$ & 26.89 & 26.5 & 2.12 & 27.29 & 12.87 \\
$\# 5$ & 9.87 & 21.1 & 0.58 & 4.62 & 7.92 \\
$\# 6$ & 25.65 & 18.1 & 2.99 & 36.35 & 12.15 \\
\hline \hline
\end{tabular}

thickness ratio between the shim and piezoelectric layers for maximum electromechanical coupling and the optimized beam aspect ratio which balances the stress applied on the beam and the total harvester volume.

\section{CONTROL OF LAYER CONFIGURATION OF HARVESTER BEAM}

A large mass is favorable for increasing the power, as seen in Eq. (1). However, an increase in mass causes the dimensions of the cantilever beam to change under a fixedfrequency condition, which is problematic in the design of piezoelectric cantilevers with the target natural frequency within a specific harvester volume. For example, as the mass increases, the length of the beam should be shortened or the thickness of the beam should be increased in order to maintain the natural frequency of the beam. ${ }^{27}$ The former approach involves a decrease in the piezoelectric active area and the latter may have an adverse effect on the power density due to an increase in beam volume and a decrease in bending deflection. In Eq. (6), an increase in $m_{e q}$ necessitates an increase in $k_{e q}$ to maintain the natural frequency and the $E I$ should be enhanced to increase $k_{e q}$ without changing the length of the beam. Thus, two technical issues should be addressed in order to enhance the output power by increasing the mass for a given beam dimension: (1) how to increase $E I$ without changing the dimensions of the beam and (2) how to prevent the crack formation in piezoelectric layers due to increase in $m_{e q}$.

To address the above issues, we considered distributed inactive layers as shown in Fig. 6(b) where two inactive layers with thickness $t_{I 1}$ were incorporated on the top and bottom surfaces of the active layers and one additional inactive layer with thickness $t_{I 2}$ was located between the two active layers. The total thickness of the inactive layers in Fig. 6(b) was same as that in Fig. 6(a). In this case, the bending stiffness of this structure can be derived as

$$
\begin{aligned}
E I= & \frac{2 b}{3}\left[c _ { I } \left(\left(\frac{t_{I 2}}{2}+2 t_{E}+t_{A}+t_{I 1}\right)^{3}-\left(\frac{t_{I 2}}{2}+2 t_{E}+t_{A}\right)^{3}\right.\right. \\
& \left.+\left(\frac{t_{I 2}}{2}\right)^{3}\right)+c_{A}\left(\left(\frac{t_{I 2}}{2}+t_{E}+t_{A}\right)^{3}-\left(\frac{t_{I 2}}{2}+t_{E}\right)^{3}\right) \\
& +c_{E}\left(\left(\frac{t_{I 2}}{2}+2 t_{E}+t_{A}\right)^{3}-\left(\frac{t_{I 2}}{2}+t_{E}+t_{A}\right)^{3}\right. \\
& \left.\left.+\left(\frac{t_{I 2}}{2}+t_{E}\right)^{3}-\left(\frac{t_{I 2}}{2}\right)^{3}\right)\right]
\end{aligned}
$$


(a)

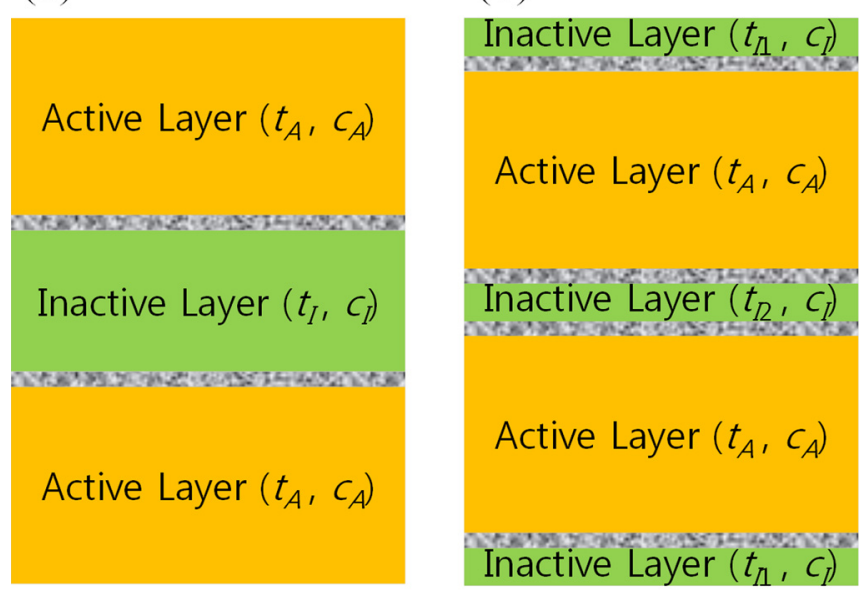

\section{3roxy Bonding Layer $\left(t_{E}, c_{E}\right)$}

FIG. 6. Layer structures of bimorph piezoelectric harvesters: (a) conventional bimorph with central inactive layer and (b) bimorph with distributed inactive layers. The total thickness of the inactive layers in (b) is the same as in (a), i.e., $t_{I}=2 t_{I 1}+t_{I 2}$.

Since the practical value of $t_{E}$ was found to be approximately $10 \mu \mathrm{m}$, the difference between the total beam thickness of the two structures in Fig. 6 is not significant. Equation (7) becomes close to Eq. (3) when the $t_{I 1}$ value becomes 0 . The only difference between Eqs. (3) and (7) when $t_{I 1}=0$ is due to the existence of the top and bottom epoxy layers in Fig. 6(b). However, their effect on the total bending stiffness is negligible, because their thickness and elastic modulus are very small compared to those of the active and inactive layers.

Considering optimized dimensions and material parameters of sample \#3 in Sec. II, we calculated the EI with respect to the ratio of elastic modulus between inactive and active layers $\left(c_{I} / c_{A}\right)$ under various $2 t_{I 1} /\left(2 t_{I 1}+t_{I 2}\right)$ conditions as shown in Fig. 7(a). The $c_{A}$ and $c_{E}$ were fixed to $66 \mathrm{GPa}$ and $24.1 \mathrm{GPa}$, respectively, for the $E I$ vs. $c_{I} / c_{A}$ plot. Note that $2 t_{I 1} /\left(2 t_{I 1}+t_{I 2}\right)=0$ corresponds to the conventional bimorph with a central inactive layer in Fig. 6(a), and the other conditions are for bimorphs with distributed inactive layers shown in Fig. 6(b). The EI has a tendency to increase linearly in proportion to the $c_{I} / c_{A}$ ratio, and the slope of this curve is enhanced with increasing $2 t_{I 1} /\left(2 t_{I 1}+t_{I 2}\right)$ value. It can be clearly seen from Fig. 7(a) that the EI of the bimorph with the distributed inactive layers is always larger than that of the bimorph with a central inactive layer once the $c_{I} / c_{A}$ ratio exceeds 1 . Therefore, the beam bending stiffness can be enhanced within the same beam volume by forming outer inactive layers with a larger elastic modulus than that of the active layer.

In Sec. II, a common spring steel was utilized that has a high elastic modulus of $210 \mathrm{GPa}$ as an inactive layer material. Therefore, the $c_{I} / c_{A}$ ratio was 3.18 , for which Fig. 7(b) shows the $E I$ vs. $2 t_{I 1} /\left(2 t_{I 1}+t_{I 2}\right)$ plot. The $E I$ gradually increases with increasing $2 t_{I 1} /\left(2 t_{I 1}+t_{I 2}\right)$ ratio from $9.99 \times 10^{-3} \mathrm{~N} \mathrm{~m}^{2}$ for the conventional bimorph to $22.27 \times 10^{-3} \mathrm{~N} \mathrm{~m}^{2}$ for $2 t_{I 1} /\left(2 t_{I 1}+t_{I 2}\right)=1$, which corresponds to the structure consisting of only outer inactive layers without the center shim. Note again that the total EI is dominated by the elastic modulus ratio between the outer inactive layer and the active layer materials, and the effect of the center shim on the EI is almost negligible, as shown in Fig. 7(c). Therefore, the selection of the center shim material does not seriously affect the elastic properties of the cantilever once the $c_{I} / c_{A}$ and $2 t_{I 1} /\left(2 t_{I 1}+t_{I 2}\right)$ conditions are fixed.

In order to confirm the effect of EI control on the real output power of the harvester experimentally, we fabricated the piezoelectric bimorph cantilevers with dimensions
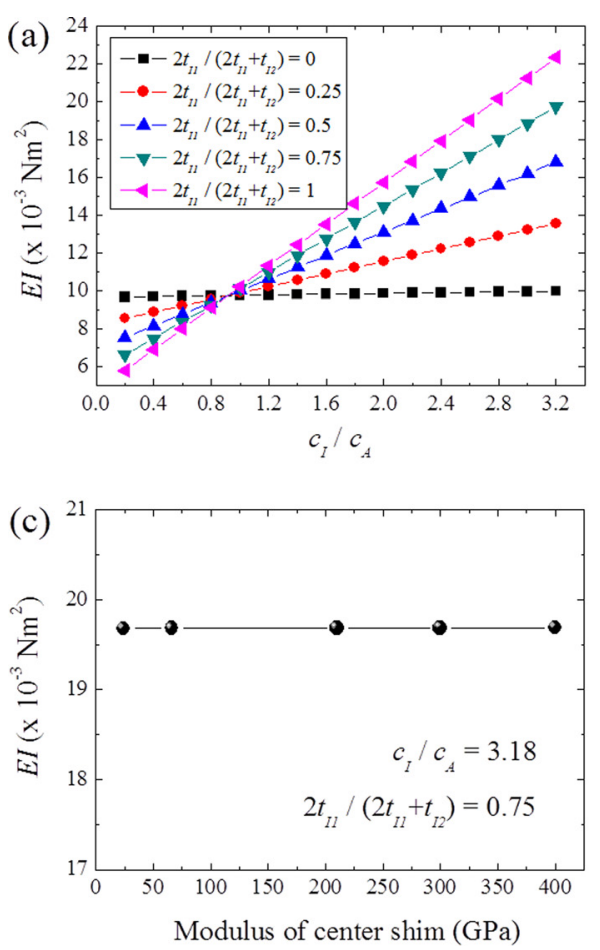

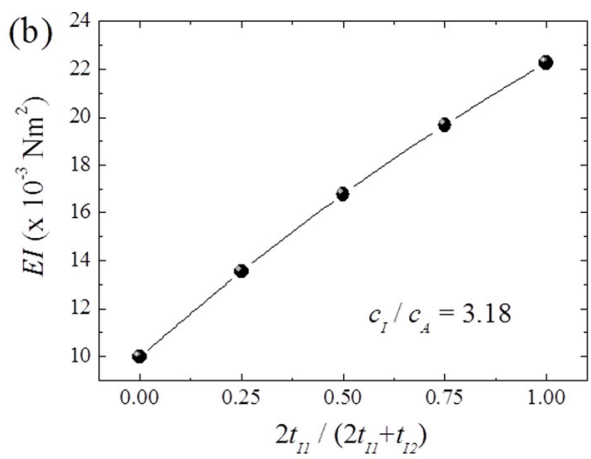

FIG. 7. Bending stiffness of the bimorph with distributed inactive layers (a) as a function of $c_{I} / c_{A}$ ratio with various $2 \mathrm{t}_{I 1} /\left(2 \mathrm{t}_{I 1}+\mathrm{t}_{I 2}\right)$ conditions, (b) as a function of $2 \mathrm{t}_{11} /\left(2 \mathrm{t}_{11}+\mathrm{t}_{12}\right)$ ratio with a constant $c_{I} / c_{A}$ of 3.18 , and (c) as a function of elastic modulus of the center shim with a constant $2 \mathrm{t}_{I 1} /\left(2 \mathrm{t}_{I 1}+\mathrm{t}_{I 2}\right)$ of 0.75 and $c_{I} / c_{A}$ of 3.18 . 


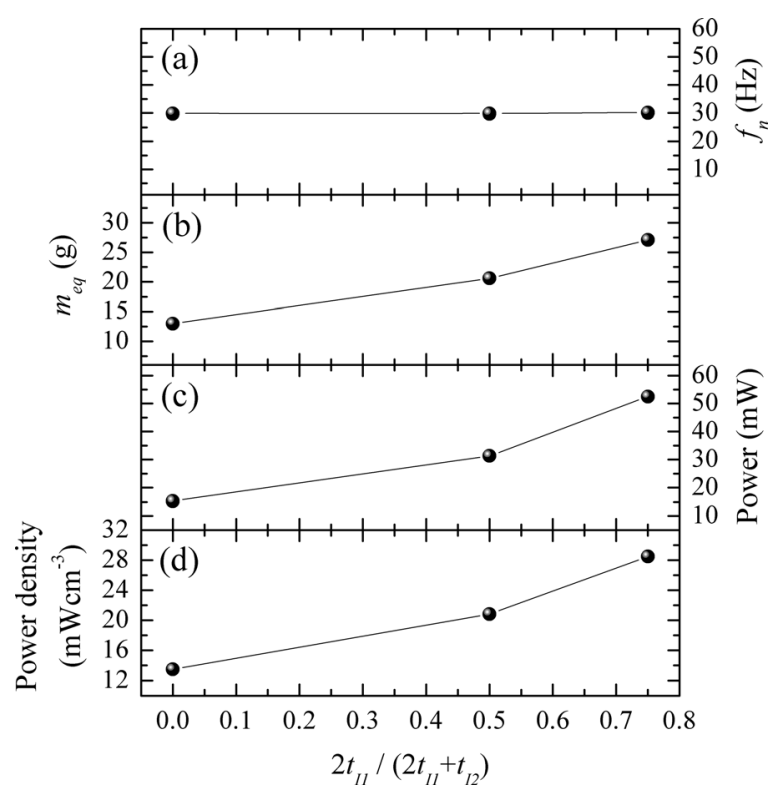

FIG. 8. (a) Measured fundamental frequency, (b) equivalent mass, (c) measured power, and (d) measured power density of the bimorph cantilevers with distributed inactive layers as a function of $2 \mathrm{t}_{I 1} /\left(2 \mathrm{t}_{I 1}+\mathrm{t}_{I 2}\right)$ ratio with a constant $c_{I} / c_{A}$ of 3.18

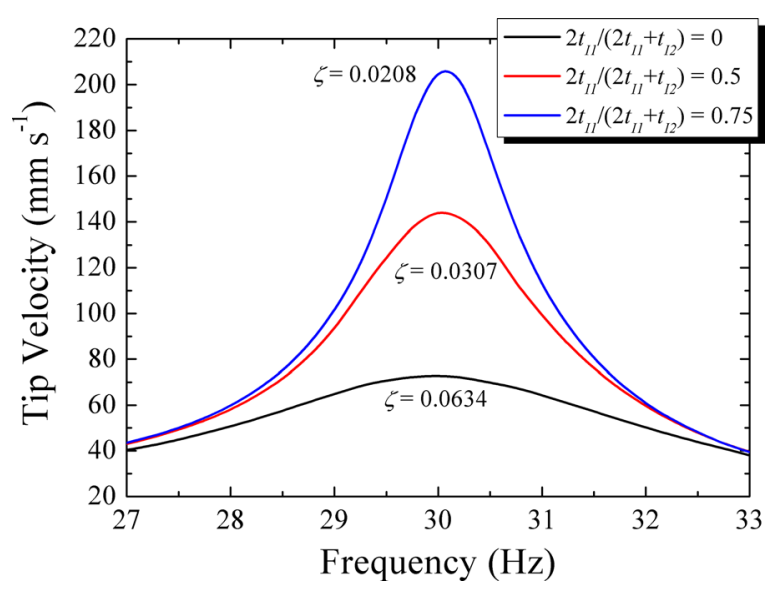

FIG. 9. Tip velocity of the bimorph cantilevers with distributed inactive layers as a function of vibration frequency.

similar to sample \#3 $(L=31 \mathrm{~mm}$ and $b=36 \mathrm{~mm})$. Three different $2 t_{I 1} /\left(2 t_{I 1}+t_{I 2}\right)$ ratios of $0,0.5$, and 0.75 were selected for comparison, and the $2 t_{I 1}+t_{I 2}$ value (total thickness of inactive layers) was fixed at $80 \mu \mathrm{m}$ in all cases. Tungsten was used as a tip mass material for the cantilevers, and its mass was optimized such that the bending resonance frequency of the cantilevers was near $30 \mathrm{~Hz}$, as shown in Fig. 8(a). The equivalent mass of the cantilever increased with increasing $2 t_{I 1} /\left(2 t_{I 1}+t_{I 2}\right)$ ratio, with values of $12.9 \mathrm{~g}$,
$20.6 \mathrm{~g}$, and $27.1 \mathrm{~g}$ for $2 t_{I 1} /\left(2 t_{I 1}+t_{I 2}\right)=0,0.5$, and 0.75 , respectively (Fig. 8(b)). Next, we measured the output power of the cantilevers. The measurement method and conditions were the same as those in Sec. II and the results are shown in Figs. 8(c) and 8(d). As the equivalent mass of the cantilever was increased, the output power and power density also increased from $15.3 \mathrm{~mW}$ and $13.47 \mathrm{~mW} \mathrm{~cm}^{-3}$ for the $2 t_{I 1} /\left(2 t_{I 1}+t_{I 2}\right)=0$ case (sample \#3) up to $52.5 \mathrm{~mW}$ and $28.48 \mathrm{~mW} \mathrm{~cm}^{-3}$ for the $2 t_{I 1} /\left(2 t_{I 1}+t_{I 2}\right)=0.75$ case. This result clearly shows that the enhancement of the bending stiffness due to the distributed inactive layers is very effective in increasing the equivalent mass under fixed-frequency conditions and consequently in enhancing the output power and power density without any significant change in the cantilever dimensions. Moreover, owing to ductile and elastic nature of the spring steel, the surfaces of harvester beam are resilient and resistant to bending stress providing additional protection to the inner active layers.

Another notable phenomenon was observed from the results in Fig. 8. According to Eq. (1), the power of the harvester is directly proportional to the equivalent mass under fixed frequency and acceleration conditions. However, the power increased more rapidly than the equivalent mass with increasing $2 t_{I 1} /\left(2 t_{I 1}+t_{I 2}\right)$, indicating that there is an additional effect of the increase in bending stiffness. Therefore, we considered the effect of other term in Eq. (1), the damping ratio $\zeta$. Since the damping ratio is given as $\zeta=c / 2 \sqrt{m_{e q} k_{e q}}$, where $c$ is the damping constant and $k_{e q}=3 E I / L^{3}$, it is closely related to the bending stiffness. Figure 9 shows the tip velocity vs. vibration frequency curve for the bimorph cantilevers as measured using a laser vibrometer (OFV-5000, Polytec) under the same acceleration of $1 \mathrm{~m} \mathrm{~s}^{-2}$. The damping ratio obtained from the resonance peak for the $2 t_{I 1} /\left(2 t_{I 1}+t_{I 2}\right)=0.75$ sample was 0.0208 , while the $2 t_{I 1} /\left(2 t_{I 1}+t_{I 2}\right)=0$ sample showed a much higher value of 0.0634 . Since the enhancement in the bending stiffness results in increase in both $m_{e q}$ and $k_{e q}$, the damping ratio can be decreased, leading to an additional increase in the output power.

The physical parameters and performance characteristics of the vibration harvesters with three different $2 t_{I 1} /\left(2 t_{I 1}+t_{I 2}\right)$ ratios are summarized in Table III. The maximum input power values calculated using Eq. (1) were higher than the measured output values, so the conversion efficiencies were $0.58,0.36$ and 0.32 for the $2 t_{I 1} /\left(2 t_{I 1}+t_{I 2}\right)$ $=0,0.5$ and 0.75 samples, respectively. This result is consistent with the observation in a prior study that the behavior of the output power is opposite to that of the efficiency with respect to the damping ratio. ${ }^{28}$ This is the reason why the conversion efficiency cannot solely represent the performance of harvesters, and the actual power or power density is

TABLE III. Physical parameters, calculated input power, measured output power, and efficiency values of piezoelectric harvesters with different $2 \mathrm{t}_{I 1} /\left(2 \mathrm{t}_{I 1}+\mathrm{t}_{I 2}\right)$ ratios.

\begin{tabular}{lcccccc}
\hline \hline $2 \mathrm{t}_{I 1} /\left(2 \mathrm{t}_{I 1}+\mathrm{t}_{I 2}\right)$ & $f_{n}(\mathrm{~Hz})$ & $Y(\mathrm{~mm})$ & $m_{\text {eq }}(\mathrm{g})$ & $\zeta$ & $P_{\text {calculated }}(\mathrm{mW})$ & $P_{\text {measured }}(\mathrm{mW})$ \\
\hline 0 & 29.8 & 0.28 & 12.986 & 0.0634 & 26.264 & 15.3 \\
0.5 & 29.8 & 0.28 & 20.597 & 0.0307 & 86.033 & 31.36 \\
0.75 & 30.1 & 0.274 & 27.107 & 0.0208 & 165.45 & 0.36 \\
\hline \hline
\end{tabular}


more useful for characterizing the device performance. Our harvester design with $2 t_{I 1} /\left(2 t_{I 1}+t_{I 2}\right)=0.75$ showed a high output power of $52.5 \mathrm{~mW}$ at $30 \mathrm{~Hz}$ under $6.9 \mathrm{~m} \mathrm{~s}^{-2}(0.7 \mathrm{G})$ acceleration, which represents a power density of 28.48 $\mathrm{mW} \mathrm{cm}{ }^{-3}$ and $58.12 \mathrm{~mW} \mathrm{~cm}^{-3} \mathrm{~g}^{-2}$ (the power density per unit square of the acceleration). To the best of our knowledge, these are the highest values reported to date in vibration energy harvesting experiments conducted at low frequencies under sub-1G acceleration. This result is very promising for powering wireless sensor nodes.

\section{SUMMARY}

We designed cantilever-type piezoelectric energy harvesters with various beam aspect ratios and investigated their power generation performance at a constant acceleration and frequency of vibration. It was found that the output power density of the harvester is optimized when the beam shape is close to square, in both constant bending stiffness (i.e., constant beam width) condition and constant beam area condition. After optimizing the beam dimensions, we investigated the effect of controlling the bending stiffness on the output power of a piezoelectric bimorph cantilever. By forming outer inactive layers with higher elastic moduli than that of the active layer, the bending stiffness could be enhanced without significantly changing the cantilever beam dimensions. The enhanced bending stiffness induced an increase in the equivalent mass and a decrease in the damping ratio, both of which contributed to an increase in power under fixed frequency and acceleration conditions. We believe that the results of this study can provide design guidance toward fabricating high power piezoelectric energy harvesters.

\section{ACKNOWLEDGMENTS}

The authors gratefully acknowledge the financial support from the Samsung GRO program.

${ }^{1}$ R. Andosca, T. G. McDonald, V. Genova, S. Rosenberg, J. Keating, C. Benedixen, and $\mathrm{J}$. Wu, "Experimental and theoretical studies on MEMS piezoelectric vibrational energy harvesters with mass loading," Sens. Actuators A 178, 76-87 (2012).

${ }^{2}$ R. Torah, P. Glynne-Jones, M. Tudor, T. O’Donnell, S. Roy and S. Beeby, "Self-powered autonomous wireless sensor node using vibration energy harvesting," Meas. Sci. Technol. 19, 125202 (2008).

${ }^{3}$ B. Marinkovic and H. Koser, "Smart sand-A wide bandwidth vibration energy harvesting platform,” Appl. Phys. Lett. 94, 103505 (2009).

${ }^{4}$ H.-B. Fang, J.-Q. Liu, Z.-Y. Xu, L. Dong, L. Wang, D. Chen, B.-C. Cai, and Y. Liu, "Fabrication and performance of MEMS-based piezoelectric power generator for vibration energy harvesting," Microelectron. J. 37, 1280-1284 (2006).

${ }^{5}$ Y. B. Jeon, R. Sood, J.-h. Jeong, and S.-G. Kim, "MEMS power generator with transverse mode thin film PZT," Sens. Actuators A 122, 16-22 (2005).

${ }^{6}$ M.-H. Seo, D.-H. Choi, I.-H. Kim, H.-J. Jung, and J.-B. Yoon, "Multiresonant energy harvester exploiting high-mode resonances frequency downshifted by a flexible body beam," Appl. Phys. Lett. 101, 123903 (2012).
${ }^{7}$ S. P. Beeby, M. J. Tudor, and N. M. White, "Energy harvesting vibration sources for microsystems applications," Meas. Sci. Technol. 17, R175-R195 (2006).

${ }^{8}$ J. C. Park, S. Khym, and J. Y. Park, "Micro-fabricated lead zirconate titanate bent cantilever energy harvester with multi-dimensional operation," Appl. Phys. Lett. 102, 043901 (2013).

${ }^{9}$ S. R. Anton and H. A. Sodano, "A review of power harvesting using piezoelectric materials (2003-2006)," Smart Mater. Struct. 16, R1-R21 (2007).

${ }^{10}$ C.-H. Choi, I.-T. Seo, D. Song, M.-S. Jang, B.-Y. Kim, S. Nahm, T.-H. Sung, and H.-C. Song, "Relation between piezoelectric properties of ceramics and output power density of energy harvester," J. Eur. Ceram. Soc. 33, 1343-1347 (2013).

${ }^{11}$ M. A. Karami and D. J. Inman, "Powering pacemakers from heartbeat vibrations using linear and nonlinear energy harvesters," Appl. Phys. Lett. 100, 042901 (2012).

${ }^{12}$ D. Shen, J.-H. Park, J. H. Noh, S.-Y. Choe, S.-H. Kim, H. C. Wikle III, and D.-J. Kim, "Micromachined PZT cantilever based on SOI structure for low frequency vibration energy harvesting," Sens. Actuators A 154, 103-108 (2009).

${ }^{13} \mathrm{Y}$. Uzun and E. Kurt, "The effect of periodic magnetic force on a piezoelectric energy harvester," Sens. Actuators A 192, 58-68 (2013.

${ }^{14}$ S. Zhou, J. Cao, A. Erturk, and J. Lin, "Enhanced broadband piezoelectric energy harvesting using rotatable magnets," Appl. Phys. Lett. 102, 173901 (2013).

${ }^{15}$ N. S. Hudak and G. G. Amatucci, "Small-scale energy harvesting through thermoelectric, vibration, and radiofrequency power conversion," J. Appl. Phys. 103, 101301 (2008).

${ }^{16}$ R. C. Kambale, W.-H. Yoon, D.-S. Park, J.-J. Choi, C.-W. Ahn, J.-W. Kim, B.-D. Hahn, D.-Y. Jeong, B. C. Lee, G.-S. Chung, and J. Ryu, "Magnetoelectric properties and magnetomechanical energy harvesting from stray vibration and electromagnetic wave by $\mathrm{Pb}\left(\mathrm{Mg}_{1 / 3} \mathrm{Nb}_{2 / 3}\right) \mathrm{O}_{3^{-}}$ $\mathrm{Pb}(\mathrm{Zr}, \mathrm{Ti}) \mathrm{O}_{3}$ single crystal/Ni cantilever," J. Appl. Phys. 113, 204108 (2013).

${ }^{17}$ S. Y. Wang, K. Tai, and S. T. Quek, "Topology optimization of piezoelectric sensors/actuators for torsional vibration control of composite plates," Smart Mater. Struct. 15, 253-269 (2006).

${ }^{18}$ B. Zheng, C.-J. Chang, and H. C. Gea, "Topology optimization of energy harvesting devices using piezoelectric materials," Struct. Multidisc. Optim. 38, 17-23 (2009).

${ }^{19}$ M. I. Friswell and S. Adhikari, "Sensor shape design for piezoelectric cantilever beams to harvest vibration energy," J. Appl. Phys. 108, 014901 (2010).

${ }^{20} \mathrm{~A}$. M. Wickenheiser, "Design optimization of linear and non-linear cantilevered energy harvesters for broadband vibrations," J. Intel. Mater. Syst. Struct. 22, 1213-1225 (2011).

${ }^{21} \mathrm{Q}$. Wang and N. Wu, "Optimal design of a piezoelectric coupled beam for power harvesting," Smart Mater. Struct. 21, 085013 (2012).

${ }^{22} \mathrm{D}$. Guyomar and M. Lallart, "Recent progress in piezoelectric conversion and energy harvesting using nonlinear electronic interfaces and issues in small scale implementation," Micromachines 2, 274-294 (2011).

${ }^{23}$ G. K. Ottman, H. F. Hofmann, A. C. Bhatt, and G. A. Lesieutre, "Adaptive piezoelectric energy harvesting circuit for wireless remote power supply," IEEE Trans. Power Electron. 17, 669-676 (2002).

${ }^{24}$ J. Yi, F. Su, Y.-H. Lam, W.-H. Ki, and C.-Y. Tsui, “An energy-adaptive MPPT power management unit for micro-power vibration energy harvesting," in Proceedings of IEEE International Symposium on Circuits and Systems (2008), pp. 2570-2573.

${ }^{25}$ Energy Harvesting Technologies, edited by S. Priya and D. J. Inman (Springer, New York, 2009)

${ }^{26} \mathrm{H}$. Hosaka, K. Itao, and S. Kuroda, "Damping characteristics of beamshaped micro-oscillators," Sens. Actuators A 49, 87-95 (1995).

${ }^{27}$ S. Roundy, E. S. Leland, J. Baker, E. Carleton, E. Reilly, E. Lai, B. Otis, J. M. Rabaey, P. K. Wright, and V. Sundararajan, "Improving power output for vibration-based energy scavengers," IEEE Pervasive Comput. 4, 28-36 (2005)

${ }^{28}$ S. Roundy, P. K. Wright, and J. M. Rabaey, Energy Scavenging for Wireless Sensor Networks with Special Focus on Vibrations (Kluwer Academic Publishers, Boston, 2004). 